\title{
Interação Fármaco-Nutriente: uma revisão sistemática
}

\author{
Drug-Nutrient Interaction: a systematic review
}

\author{
Ana Júlia Carvalho Rocha ${ }^{\bullet}$, Ana Rafaela Labouré de Carvalho Vieira ${ }^{\bullet}$, André Luiz Oliveira de \\ Faria ${ }^{\bullet}$, Guilherme Henrique dos Santos Silva ${ }^{\circ}$, Gérsika Bitencourt Santos" ${ }^{\circ}$
}

Universidade José do Rosário Vellano, Alfenas, Minas Gerais, Brasil. Autor para correspondência. E-mail: gersika.santos@unifenas.br

\begin{abstract}
Resumo: Introdução: Este estudo tem como objetivo revisar os artigos científicos publicados acerca da interação fármaco - alimento. Revisão: Para o direcionamento desta pesquisa, foram selecionados os artigos publicados entre 2014 e 2021, disponibilizados em bancos de dados como PubMed, SciELO, periódicos da CAPES e Google Scholar. Para a seleção dos artigos, foi usado como critério as apresentações que demonstravam os mecanismos das interações fármacos - alimento de maneira mais sucinta ou que detinham um grupo de estudo específico. Para tal feito, foi efetuada a leitura de 97 resumos de artigos, 55 artigos lidos por completo e selecionados 20 desses para embasar essa revisão. Discussão: Sendo assim, pode-se extrair dessa revisão a grande importância de deter o conhecimento dessas interações, visto que foi averiguada na totalidade das publicações lidas a recorrência dessas interações e o quanto pode ser maléfica para o paciente caso diminua a efetividade do tratamento. Considerações finais: Dessa forma, ao conhecer as principais interações medicamentosas é possível direcionar o paciente a uma terapêutica eficiente, sem conter relação fármaco-nutriente prejudicial, e, consequentemente, aumentar a efetividade do tratamento e reduzir eventuais danos colaterais.
\end{abstract}

Palavras-chave: drogas, interações farmacológicas, nutrientes.

\begin{abstract}
Introduction: This study aims to review the published scientific articles about the drug - food interaction. Review: For the purpose of this research, articles published between 2014 and 2021 were selected, made available in databases such as PubMed, sciELO, CAPES journals and Google Scholar. For the selection of articles, presentations that demonstrated the mechanisms of drug - food interactions in a more succinct manner or that held a specific study group were used as criteria. For this purpose, 97 article abstracts were read, 55 articles were read in full and 20 of them were selected to support this review. Discussion: Thus, it is possible to extract from this review the great importance of having knowledge of these interactions, since it was ascertained in all publications read the recurrence of these interactions and how harmful they can be to the patient if the effectiveness of the treatment decreases. Final considerations: Thus, by knowing the main drug interactions, it is possible to direct the patient to an efficient therapy, without containing a harmful drug-nutrient relationship, and, consequently, increase the effectiveness of the treatment and reduce eventual collateral damage.
\end{abstract}

Keywords: drugs, pharmacological interactions, nutrient.

\section{Introdução}

Interações medicamentosas são interferências no efeito farmacológico devido a alguma substância; podendo gerar efeitos benéficos ou maléficos. A interação fármaco-nutriente ocorre diante da influência tanto do fármaco com a absorção do nutriente ingerido quanto do nutriente na ação e eficácia do fármaco (Dantas et al., 2018).

A relevância das interações fármaco-nutrientes é notável tanto no ambiente hospitalar quanto fora dele pela persistência de subavaliações, negligências e ignorância das interações nutricionais. Por isso, é de extrema importância o entendimento da influência mútua do fármaco com a alimentação como um todo pela equipe hospitalar, englobando todas as áreas do local e a orientação aos pacientes e familiares para que os efeitos desejados ocorram (Leal \& Júnior, 2018).

Ao se administrar um medicamento, este interage com o organismo de duas maneiras: a farmacodinâmica e a farmacocinética. A farmacodinâmica consiste na ação da medicação no organismo, enquanto a farmacocinética compreende as etapas de sua disposição pelo corpo, sendo elas: absorção, distribuição, metabolização e excreção. É sabido que a ingestão de alguns alimentos ou sua falta podem alterar essas etapas, assim como o uso de medicamentos pode modificar a absorção de certos nutrientes. Diante disso, observa-se que a combinação fármaco-nutriente de maneira errada pode comprometer a clínica do paciente (Macedo et al., 2020). 
Este estudo tem como objetivo realizar uma revisão sistemática sobre as interações fármaco-nutrientes destacando sua definição, relevância e ocorrência em diversos níveis hospitalares e em ambiente não hospitalar.

\section{Revisão}

Para realização desta revisão sistemática foram realizadas buscas de artigos científicos nos seguintes bancos de dados: National Library of Medicine (PubMED), ScientificElectronic Library Online (SciELO) e periódicos da CAPES. Para o direcionamento dessa revisão os descritores para essa busca foram: "Interações fármacos-nutrientes”, “Interações entre medicamentos e alimentos” e “Drug-nutrientinteractions”.

Foram utilizados como critério para escolha dos artigos aqueles que demonstraram os mecanismos das interações fármaco - nutrientes de maneira mais sucintas, soma-se a isso também, para essa escolha, a averiguação dos grupos de estudos que os artigos direcionaram suas pesquisas e a ocorrência dessas interações nos grupos. Vale ressaltar que a busca foi direcionada para artigos a partir de 2014 (2014 - 2021). Após a aplicação dos critérios de escolha, para a execução dessa revisão sistemática, foram lidos 97 resumos; 55 artigos por completo e selecionados 20 artigos para incorporação dessa revisão, conforme demonstrado na tabela (Tabela 1).

Tabela 1. Apresentação das referências bibliográficas apanhados através das bases de dados do Google Scholar, PubMed, SciELO e portal CAPES.

\begin{tabular}{|c|c|c|c|c|c|}
\hline $\begin{array}{l}\text { Bases de } \\
\text { Dados }\end{array}$ & Descritores Utilizados & $\begin{array}{l}\text { Número de } \\
\text { Referências } \\
\text { Obtidas }\end{array}$ & $\begin{array}{l}\text { Resumo } \\
\text { Analisados }\end{array}$ & $\begin{array}{l}\text { Referências } \\
\text { Selecionadas } \\
\text { para Análise }\end{array}$ & $\begin{array}{l}\text { Selecionados } \\
\text { para Revisão }\end{array}$ \\
\hline \multirow{3}{*}{$\begin{array}{l}\text { Google } \\
\text { Scholar }\end{array}$} & Interação fármaco-nutriente & 13.800 & 10 & 6 & 3 \\
\hline & $\begin{array}{l}\text { Interação entre medicamentos } \\
\text { e alimentos }\end{array}$ & 15.200 & 8 & 3 & 1 \\
\hline & Drug-nutrient interactions & 1.800 & 10 & 3 & 1 \\
\hline \multirow{3}{*}{ PubMed } & Interação fármaco- nutriente & 79 & 20 & 12 & 6 \\
\hline & $\begin{array}{l}\text { Interação entre medicamentos } \\
\text { e alimentos }\end{array}$ & 0 & 0 & 0 & 0 \\
\hline & Drug-nutrient interactions & 1.342 & 20 & 13 & 3 \\
\hline \multirow{3}{*}{ SciELO } & Interação fármaco-nutriente & 4 & 4 & 3 & 3 \\
\hline & $\begin{array}{l}\text { Interação entre medicamentos } \\
\text { e alimentos }\end{array}$ & 1 & 1 & 1 & 0 \\
\hline & Drug-nutrient interactions & 1 & 1 & 1 & 1 \\
\hline \multirow{3}{*}{ CAPES } & Interação fármaco-nutriente & 7 & 4 & 3 & 1 \\
\hline & $\begin{array}{l}\text { Interação entre medicamentos } \\
\text { e alimentos }\end{array}$ & 48 & 10 & 6 & 0 \\
\hline & Drug-nutrient interactions & 1.439 & 9 & 4 & 1 \\
\hline
\end{tabular}

Dentre os 20 artigos analisados publicados entre os anos de 2014 e 2020, 12 deles são publicações internacionais e os outros 8 artigos de cenário nacional. O quadro abaixo representa as principais características das publicações selecionadas (Quadro 1).

Quadro 1. Caracterização dos artigos com relação à interação fármaco-nutrientes.

\begin{tabular}{|c|c|c|c|}
\hline Ano & Autores & Revista & Principais Achados \\
\hline 2020 & $\begin{array}{c}\text { Macedo et al., } \\
2020\end{array}$ & $\begin{array}{c}\text { Research, Society and } \\
\text { Development }\end{array}$ & $\begin{array}{c}\text { Foi feita uma revisão integrativa das interações droga-nutrientes em } \\
\text { pacientes pediátricos. A ação do medicamento pode ser alterada pelo } \\
\text { nutriente e a disponibilidade desse nutriente também pode diminuir e } \\
\text { causar efeitos colaterais. }\end{array}$ \\
\hline 2019 & $\begin{array}{c}\text { Koziolek et al., } \\
2019\end{array}$ & $\begin{array}{c}\text { European Journal of } \\
\text { Pharmaceutical } \\
\text { Sciences }\end{array}$ & $\begin{array}{c}\text { A ingestão do conjunto medicamento/alimento pode afetar a liberação } \\
\text { do fármaco (volume e composição dos fluidos luminais, tempo de } \\
\text { trânsito, motilidade), absorção (transportadores e efluxo), distribuição } \\
\text { (transporte de droga linfática, lipoproteína e proteína plasmática de } \\
\text { ligação), metabolismo e eliminação (enzimas de metabolização e } \\
\text { transportadores de drogas. }\end{array}$ \\
\hline 2019 & $\begin{array}{c}\text { Seyen et al., } \\
2019\end{array}$ & $\begin{array}{c}\text { Clinical Pharmacology } \\
\text { \& Therapeutics }\end{array}$ & $\begin{array}{c}\text { Estudos mostraram que há uma interação medicamentosa em pacientes } \\
\text { que tomam sofosbuvir / velpatasvir 400/100 mg (tratamento de } \\
\text { Hepatite C) e que tomam omeprazol. Portanto, o refrigerante de cola } \\
\text { pode usada para superar a interação droga-droga entre VEL e } \\
\text { omeprazol. }\end{array}$ \\
\hline
\end{tabular}




\begin{tabular}{|c|c|c|c|}
\hline 2018 & $\begin{array}{l}\text { Leal \& Júnior, } \\
2018\end{array}$ & Revista Rios Saúde & $\begin{array}{c}\text { Foi comprovado interações fármaco-alimentos relevantes em pacientes } \\
\text { que usam Captopril (alimentos em geral), Hidroclorotiazida (ovo, carne } \\
\text { e queijo), Ácido acetilsalicílico (Vit. C e ácido fólico), Paracetamol } \\
\text { (fibras), carbamazepina (Vit. D), 6-Mercaptopurina (Leite) e } \\
\text { Tetraciclinas (alimentos ricos em cálcio e ferro). }\end{array}$ \\
\hline 2018 & $\begin{array}{l}\text { Hermann et al., } \\
2018\end{array}$ & $\begin{array}{c}\text { The Journal of Clinical } \\
\text { Pharmacology }\end{array}$ & $\begin{array}{l}\text { Os dois principais mecanismos de interações nutrientes-droga são } \\
\text { através da farmacodinâmica e farmacocinética, que podem ter um } \\
\text { efeito sinérgico ou antagonista e até levar a um efeito não esperado. }\end{array}$ \\
\hline 2018 & $\begin{array}{l}\text { Koziolek et al., } \\
2018\end{array}$ & $\begin{array}{l}\text { Pharmaceutical } \\
\text { Research }\end{array}$ & $\begin{array}{l}\text { Os lipídios no TGI podem afetar a liberação das drogas. Em fármacos } \\
\text { lipofílicos, aumentam a absorção dessas drogas. Porém, os lipídios } \\
\text { podem afetar em mudanças do pH intestinal, a solução e taxas de } \\
\text { esvaziamento, ou por meio de mudanças a jusante para as condições } \\
\text { intestinais, diminuindo a absorção dos fármacos não lipofílicos. }\end{array}$ \\
\hline 2018 & $\begin{array}{l}\text { Mohn et al., } \\
2018\end{array}$ & Pharmaceutics & $\begin{array}{l}\text { O uso de drogas comumente prescritas pode ter um impacto adverso no } \\
\text { estado nutricional, a crescente prevalência de condições que exigem } \\
\text { uso crônico faz com que esses atrapalhem a absorçâo de nutrientes } \\
\text { essenciais, causando um potencial problema de saúde pública. }\end{array}$ \\
\hline 2018 & $\begin{array}{l}\text { Dantas et al., } \\
2018\end{array}$ & $\begin{array}{c}\text { Revista de } \\
\text { Biotecnologia \& } \\
\text { Ciência }\end{array}$ & $\begin{array}{l}\text { O uso concomitante de antiparasitários e antibacterianos com } \\
\text { alimentos causa uma diminuição e também aumento da } \\
\text { biodisponibilidade desses medicamentos. Nesse caso pode ter um efeito }\end{array}$ \\
\hline 2018 & $\begin{array}{l}\text { Ferreira et al., } \\
2018\end{array}$ & $\begin{array}{c}\text { International Journal of } \\
\text { Nutrology }\end{array}$ & $\begin{array}{l}\text { As proteínas são quebradas em aminoácidos no intestino e utilizam o } \\
\text { mesmo mecanismo de transporte na corrente sanguínea que o } \\
\text { medicamento Levodopa. Com isso, pacientes que tomam esse } \\
\text { antiparkinsoniano não podem fazer muita ingestão de proteínas e nem } \\
\text { tomar o remédio concomitantemente com as refeições. }\end{array}$ \\
\hline 2018 & $\begin{array}{l}\text { Oliveira et al., } \\
2018\end{array}$ & $\begin{array}{c}\text { International Journal of } \\
\text { Nutrology }\end{array}$ & $\begin{array}{l}\text { As tetraciclinas quando administradas com leites e derivados } \\
\text { (alimentos que contém cálcio) pode provocar uma quelação dos cátions } \\
\text { tornando-os insolúveis e não sendo absorvíveis pelo trato } \\
\text { gastrointestinal, além de diminuir também a absorção do fármaco. }\end{array}$ \\
\hline 2017 & $\begin{array}{l}\text { Amadi \& } \\
\text { Mgbahurike, } \\
2017\end{array}$ & $\begin{array}{l}\text { American Journal of } \\
\text { Therapeutics }\end{array}$ & $\begin{array}{l}\text { Comprovou- se que alguns nutrientes, ervas, chás provocam alteração } \\
\text { no esvaziamento gástrico, alteração no pH gastrointestinal, } \\
\text { complexação de drogas com a dieta, induz/inibe enzimas. É importante } \\
\text { que os profissionais avaliem adequadamente o padrão alimentar dos } \\
\text { pacientes. }\end{array}$ \\
\hline 2017 & Zha, 2017 & $\begin{array}{l}\text { Journal of Food and } \\
\text { Drug Analysis }\end{array}$ & $\begin{array}{l}\text { O uso crescente de produtos naturais em pacientes cardiovasculares } \\
\text { (CV) tem levantado preocupações sobre as potenciais interações. } \\
\text { Alguns desses podem levar a efeitos adversos cardiovasculares } \\
\text { inesperados e é, portanto, essencial para identificar ou prever } \\
\text { potenciais interações medicamentosas do produto natural com CV, e } \\
\text { compreender os mecanismos subjacentes. }\end{array}$ \\
\hline 2017 & $\begin{array}{l}\text { Minno et al., } \\
2017\end{array}$ & Blood Reviews & $\begin{array}{l}\text { Os anticoagulantes orais antagonistas de vitamina K são amplamente } \\
\text { utilizado em todo o mundo, porém esses medicamentos causam reações } \\
\text { adversas com muitos alimentos, bebidas e com outros medicamentos. } \\
\text { Com isso, estão sendo substituídos pelos Anticoagulantes Orais } \\
\text { Diretos, que causam menos interações. }\end{array}$ \\
\hline 2017 & $\begin{array}{l}\text { Deng et al., } \\
2017\end{array}$ & Drugs & $\begin{array}{l}\text { O efeito alimentar é um fenômeno comum para a administração das } \\
\text { drogas filtradas. Os efeitos têm muitos mecanismos fisiológicos que } \\
\text { contribui diferentemente em cada medicamento e em cada indivíduo. } \\
\text { Por isso, é essencial conhecer as propriedades de cada fármaco antes de } \\
\text { receita-lo. }\end{array}$ \\
\hline 2016 & $\begin{array}{l}\text { Bailey et al., } \\
2016\end{array}$ & $\begin{array}{c}\text { American Journal of } \\
\text { Hypertension, }\end{array}$ & $\begin{array}{c}\text { A ingestão intermitente de café pode complicar o diagnóstico de } \\
\text { hipertensão arterial e gestão para muitos indivíduos, pois o café tem } \\
\text { efeitos no organismo e também quando está sendo tomado junto com } \\
\text { medicamentos anti-hipertensivos. }\end{array}$ \\
\hline 2016 & $\begin{array}{c}\text { Bago \& Culig, } \\
2016\end{array}$ & Clinical Therapeutics & $\begin{array}{l}\text { Alguns alimentos em específicos foram selecionados que mais ocorrem } \\
\text { alterações quando consumidos juntamente com medicamentos. São } \\
\text { exemplos deles: Toranja, suco de laranja, vegetais em folhas, bebidas } \\
\text { contendo xantina (café, chá), tiramina (chocolate, queijo, vinho) e leite. }\end{array}$ \\
\hline 2014 & Reis et al., 2014 & $\begin{array}{l}\text { Revista Brasileira de } \\
\text { Enfermagem }\end{array}$ & $\begin{array}{l}\text { Foram analisados pacientes que faziam uso de nutrição enteral e que } \\
\text { estavam na UTI sendo medicados. Diante da pesquisa conclui-se que os } \\
\text { pacientes sofreram interações fármaco-nutrição enteral e com isso, a } \\
\text { equipe deve elaborar um protocolo para administração desses } \\
\text { medicamentos citados no estudo nesses tipos de paciente. }\end{array}$ \\
\hline 2014 & $\begin{array}{l}\text { Lombardo \& } \\
\text { Eserian, } 2014\end{array}$ & $\begin{array}{l}\text { Infarma Ciências } \\
\text { Farmacêuticas }\end{array}$ & $\begin{array}{l}\text { Os aminoácidos podem inibir a absorção de fármacos (levodopa e } \\
\text { metildopa), as fibras podem reduzir a absorção da digoxina. Dieta } \\
\text { hiperlipídica pode aumentar a absorção e trazer riscos, a exemplo da } \\
\text { carbamazepina ou mesmo ser requerida para favorecer a absorção, } \\
\text { como a griseofulvina. O pH da dieta também pode reduzir a eficácia. }\end{array}$ \\
\hline
\end{tabular}




\begin{tabular}{|c|c|c|c|}
\hline 2014 & $\begin{array}{c}\text { Antunes \& } \\
\text { Prete, 2014 }\end{array}$ & $\begin{array}{c}\text { Infarma Ciências } \\
\text { Farmacêuticas }\end{array}$ & $\begin{array}{c}\text { Os testes de biodisponibilidade feitos pela indústria farmacêutica são } \\
\text { realizados com os pacientes em jejum por exigência dos órgãos } \\
\text { sanitários. Diante disso, os profissionais não conhecem tantas } \\
\text { interações medicamentosas com os alimentos trazendo um efeito } \\
\text { maléfico ao paciente. }\end{array}$ \\
\hline 2014 & $\begin{array}{c}\text { Silva \& Novaes, } \\
2014\end{array}$ & Nutrición Hospitalaria & $\begin{array}{c}\text { Ocorre alterações em medicamentos que são administrados juntos com } \\
\text { a nutrição enteral, como a ciprofloxacina e fenitoína (diminuição da } \\
\text { biodisponibilidade), clorpromazina (precipitação e redução da } \\
\text { biodisponibilidade), hidróxido de alumínio (precipitação e redução), } \\
\text { óleo mineral (altera absorção de vitaminas solúveis em gordura). }\end{array}$ \\
\hline
\end{tabular}

Os resultados obtidos e interpretados através da análise dos artigos escolhidos, foram unânimes ao relatar que há interações entre medicamentos e nutrientes quando estes são tomados concomitantemente. Não são todos os medicamentos e nutrientes que interagem, há alguns tipos específicos. Essa interação pode resultar na diminuição na eficácia farmacológica, ampliação ou diminuição da absorção do medicamento no trato gastrointestinal, aumento dos efeitos adversos, depleção de nutrientes específicos e alterações no estado nutricional (Leal \& Júnior, 2018).

De acordo com o quadro acima, pode-se constatar que cinco desses artigos referem a situações específicas de interação entre os nutrientes e medicações. O primeiro deles destaca a dificuldade de medicar pacientes pediátricos e com isso, muitas vezes os remédios são introduzidos junto com a alimentação com o intuito de diminuir o gosto ou a criança não perceber tal ação. Ainda, teve trabalhos que falaram sobre as interações do uso de refrigerante, além do uso de café e chás estimulantes em pacientes que usam drogas para problemas cardiovasculares, também o consumo de uma dieta rica em lipídios e de uma dieta enteral.

Mediante análise sistemática dos estudos, os outros dez artigos caracterizaram a interação fármaco-nutrientes de uma maneira geral. Observaram diversas formas que afetaram tanto o comprometimento da ação do fármaco, quanto na absorção de nutrientes para o corpo. Assim, todos os 20 artigos analisados comprovaram que há interações entre alguns nutrientes e drogas e ainda frisaram que os profissionais de saúde não conhecem e nem explicam as interações que podem causar aos pacientes, levando a um efeito indesejado.

\section{Discussão}

As análises sobre a interação fármaco-nutriente apresentaram um crescimento notável nos últimos anos. As publicações nacionais e internacionais demonstram que as interações mais significativas ocorrem quando o fármaco passa da superfície em que foi administrado para a circulação, sofrendo a influência de diversos fatores, dentre eles: alteração do $\mathrm{pH}$ do conteúdo presente no trato gastrointestinal, modificação da velocidade de esvaziamento gástrico, aumento da velocidade da passagem de alimentos durante a digestão, disputa por sítios de absorção e ligação direta do fármaco com parte integrante dos alimentos (Antunes \& Prete, 2014; Koziolek et al., 2019).

Os estudos selecionados nesta revisão sistemática demonstram duas vertentes da interação dos medicamentos com os alimentos, uma vez que esta pode ser benéfica ou maléfica dependendo da situação que se encontra o paciente. O diclofenaco, por exemplo, recomenda-se que seja ingerido com alimentos para diminuir o risco de lesão intestinal, já o paracetamol não deve ser ingerido com alimentos ricos em fibras, pois leva à diminuição da absorção do fármaco (Hermann et al., 2018).

Apesar das inúmeras propagandas veiculadas pela mídia e do intenso alerta médico sobre a automedicação, pode-se observar um elevado aumento de interações fármaco-nutrientes por causa disso. Como exemplo, pode-se citar a ivermectina, antiparasitário que tem se destacado na atual situação da pandemia mundial do COVID-19. Tal medicamento tem sido usado de maneira indiscriminada e muitas vezes sem nenhuma orientação médica; e ao ser administrado junto ou próximo a alguma refeição tem sua absorção prejudicada e consequentemente, um efeito subterapêutico (Dantas et al., 2018).

Além dos fatores supracitados, há vários outros relacionados à ocorrência de interações fármaco-alimento, como as características dos elementos, a dose, o horário de administração do medicamento e da dieta, quadro clínico, polifarmácia. Tais interações despertam maior atenção no caso de pacientes suscetíveis e hospitalizados, principalmente no uso de fármacos de estreita margem de segurança ou que necessitem ajuste de dose, pois pequenas mudanças na farmacocinética podem causar grandes interferências na eficácia e segurança do tratamento (Lombardo \& Eserian, 2014).

Sobre esse assunto é consenso na literatura que as crianças compõem um grupo etário que está a todo momento exposto à situações de complicações do seu quadro clínico devido interações droga-nutrientes. Isso 
acontece em grande parte devido a imprecisão existente sobre a eficácia e segurança dos medicamentos para o grupo pediátrico. A quase totalidade de medicamentos prescritos para crianças foram testados somente em adultos. Com muita frequência, a dificuldade em ofertar a droga leva à sua administração concomitante com a refeição, o que pode resultar em falhas no tratamento e até mesmo impactar em resultados tóxicos (Macedo et al., 2020).

Quando se fala em estado nutricional, cita-se a obesidade e a desnutrição, fatores que podem alterar a farmacocinética dos fármacos. Um exemplo clássico é a alteração da absorção, distribuição e excreção das penicilinas em pacientes obesos. Penicilinas são antibióticos hidrofílicos, eliminados pelos rins, apresentando baixa penetração intracelular e tecidual, principalmente no tecido adiposo. Dessa forma, em obesos devido à porcentagem elevada de adiposidade há aumento na eliminação renal, consequentemente, diminuindo a eficácia do tratamento e aumentando a probabilidade de lesão renal. Deve- se atentar a uma dose medicamentosa que minimize os efeitos adversos e maximize a eficiência terapêutica (Leal \& Júnior, 2018).

De acordo com estudo presente em Reis et al. (2014), a absorção da hidralazina e da fenitoína quando administrada com nutrição enteral acarreta em redução da concentração plasmática, o que pode implicar na falta do efeito anti-hipertensivo e anticonvulsivante, respectivamente. As formulações contendo soja aumentam a excreção fecal da levotiroxina, causando hipotireoidismo, principalmente em períodos de administração que superam sete dias. Estudos demonstraram que a varfarina interage mesmo em formulações com baixa quantidade de vitamina $\mathrm{K}$, mas o mecanismo ainda não é totalmente conhecido. Para reduzir os efeitos das potenciais interações fármaco-nutrientes se faz necessário organizar o horário de administração do medicamento, visando a periodicidade e o tipo de dieta.

É impressionante a quantidade e variedade de alimentos comuns presentes no cotidiano da população que interagem com algum tipo de medicamento. Seyen et al. (2019) demonstram em estudo a existência de interação medicamentosa entre fármacos utilizados no tratamento da hepatite $\mathrm{C}$, sofosbuvir $400 \mathrm{mg}$ e velpatasvir $100 \mathrm{mg}$ em indivíduos que faziam uso concomitante de omeprazol sendo superada pela ingestão paralela de refrigerante de cola.

O café é uma bebida mundial e pode aumentar agudamente a pressão arterial devido a presença da cafeína. Esse efeito foi associado com eficácia reduzida de medicamentos anti-hipertensivos. Diante da combinação cafeína e felodipina, uma possível explicação é que a vasoconstrição mediada pela cafeína modificou o efeito vasodilatador da felodipina. O aumento de pressão provocado pelo café não foi abolido pela felodipina na dose máxima recomendada de $10 \mathrm{mg}$. Ademais, o consumo de cafeína anteriormente à uma consulta médica pode levar a um diagnóstico equivocado de hipertensão arterial por alterar a hemodinâmica do organismo (Bailey et al., 2016).

Produtos naturais têm sido muito utilizados em pacientes com doenças cardiovasculares e esses, combinados com medicamentos. Todavia, essas combinações precisam ser avaliadas por um médico para não se revelar de alto risco. Um exemplo é o uso do chá verde. Considerado a bebida mais consumida no mundo é constituído de polifenóis, que possuem diversos efeitos protetores na saúde cardiovascular, no entanto, ao inibir determinados transportadores torna-se prejudicial o seu uso com alguns medicamentos presentes na terapia cardiovascular (Zha, 2017).

Estudos recentes demonstraram que os fitoquímicos podem influenciar na eficácia terapêutica de medicamentos. Por exemplo, o suco de toranja aumenta a biodisponibilidade oral da felodipina, nifedipina, saquinavir e sildenafil pela inibição da CYP3A4, que auxilia na metabolização desses medicamentos. Ademais, tem-se que algumas regiões possuem o costume de ingerir ervas medicinais e paralelamente a maior parte da população faz uso contínuo de pelo menos um medicamento. Logo, é preciso expor para os pacientes a situação, pois o medicamento e a erva só podem ser usados juntos desde que não sofram interação e se tornem prejudiciais à saúde. A falha do médico em gerenciar as interações entre alimentos e medicamentos pode levar a consequências graves. É preciso conhecer os mecanismos e otimizar a terapia (Amadi \& Mgbahurike, 2017).

\section{Considerações finais}

Tendo em vista todo o exposto, chega-se à conclusão da importância de se abordar as interações fármacosnutrientes, visto que da totalidade dos artigos estudados pode-se observar a interferência de um sobre o outro em vários casos. Diante das interações notadas no estudo, observamos principalmente o mecanismo dessas, para que assim, essas comunicações fármacos-nutrientes sejam evitadas quando maléficas e utilizadas quando benéficas. Sendo assim, observa-se como resultado dessa revisão sistemática a recorrência dessas interações e a pertinência que isso ocorre na sociedade, podendo afetar direta ou indiretamente na resposta farmacológica dos medicamentos. 


\section{References}

Amadi, C. N., \& Mgbahurike, A. A. 2017. Selected Food/Herb-Drug Interactions: Mechanisms and Clinical Relevance. American Journal of Therapeutics, 25(4), 423-433.

Antunes, A. O., \& Prete, A. C. L. 2014. O papel da atenção farmacêutica frente às interações fármaconutriente. Infarma Ciências Farmacêuticas, 26(4), 208-214.

Bailey, D. G., Dresser, G. K., Urquhart, B. L., Freeman, D. J., \& Arnold, J. M. 2016. Coffee-Antihypertensive Drug Interaction: A Hemodynamic and Pharmacokinetic Study With Felodipine. American Journal of Hypertension, 29(12), 1386-1393.

Bago, M., \& Culig, J. 2016. Food and Drug Interactions. Clinical Therapeutics, 38(10), S10.

Dantas, N. M. F., Júnior, F. P. A., dos Santos, A. K. C., \& Menezes, M. E. S. 2018. Principais interações fármaconutriente envolvendo antimicrobianos e antiparasitários. Revista de Biotecnologia \& Ciência, 7(1), 24-33.

Deng, J., Zhu, X., Chen, Z., Fan, C. H., Kwan, H. S., Wong, C. H., Shek, K. Y., Zuo, Z., \& Lam, T. N. 2017. A Review of Food-DrugInteractions on Oral DrugAbsorption. Drugs, 77(17), 1833-1855.

Ferreira, C. F. M., Lima, R. P. A., \& Pontes, E. D. 2018. Interação Fármaco-Nutriente entre Levodopa e Proteínas. International Journal of Nutrology, 11(S01): S24-S327.

Hermann, R., Derendorf, H., von Richter, O., \& Rostami-Hodjegan, A. 2018. Drug-Drug and Food-Drug Interactions. The Journal of Clinical Pharmacology.

Koziolek, M., Carrière, F., \& Porter, C. J. H. 2018. Lipids in the Stomach - Implications for the Evaluation of Food Effects on Oral Drug Absorption. Pharmaceutical Research, 35(3), 55.

Koziolek, M., Alcaro, S., Augustijns, P., Basit, A. W., Grimm, M., Hens, B., Hoad, C. L., Jedamzik, P., Madla, C. M., Maliepaard, M., Marciani, L., Maruca, A., Parrott, N., Pávek, P., Porter, C. J. H., Reppas, C., Riet-Nales, D. V., Rubbens, J., Statelova, M., Trevaskis, N. L., Valentová, K., Vertzoni, M., Čepo, D. V., \& Corsettif, M. 2019. The mechanisms of pharmacokinetic food-drug interactions - A perspective from the UNGAP group. European Journal of Pharmaceutical Sciences, 134, 31-59.

Leal, M. M. F. V., \& Júnior, J. J. S. 2018. Interações fármaco nutriente: caracterização e métodos inovadores de avaliação. Revista Rios Saúde, 1(4), 38-48.

Lombardo, M., \& Eserian, J. K. 2014. Fármacos e alimentos: interações e influências na terapêutica. Infarma Ciências farmacêuticas, 26(3), 188-192.

Macedo, J. L., Branco, C. C. F. C., Ramos, S. M. N., \& Araújo, J. M. S. 2020. Interação droga-nutriente em pacientes pediátricos hospitalizados: uma revisão integrativa. Research, Society and Development, 9(1), 67911624.

Minno, A. D., Frigerio, B., Spadarella, G., Ravani, A., Sansaro, D., Amato, M., Kitzmiller, J., Pepi, M., Tremoli, E., \& Baldassarre, D. 2017. Old and new oral anticoagulants: food, herbal medicines and drug interactions. Blood Reviews, 31(4), 193-203.

Mohn, E. S., Kern, H. J., Saltzman, E., Mitmesser, S., \& McKay, D. L. 2018. Evidence of Drug-Nutrient Interactions with Chronic Use of Commonly Prescribed Medications: An Update. Pharmaceutics, 10(1), 36.

Oliveira, M. H. M., Silva, A. P. F., Araujo, A. P. S., Pontes, E. D. S., Júnior, I. R. D., Penaforte, N. F., Costa, M. C., R. A., Santos, T. M., Moura, M. W. S., \& Melo, L. R. S. 2018. Processo de Interação Fármaco - Nutriente Entre a Tetraciclina e o Cálcio. International Journal of Nutrology, 11(S01), S24-S327.

Reis, A. M. M., Carvalho, R. E. F. L., Faria, L. M. P., Oliveira, R. C., Zago, K. S. A., Cavelagna, M. F., Silva, A. G., Neto, M. L. \& Cassiani, S. H. B. 2014. Prevalência e significância clínica de interações fármaco-nutrição enteral em Unidades de Terapia Intensiva. Revista Brasileira de Enfermagem, 67(1), 85-90.

Silva, R. F., \& Novaes, M. R. C. G. 2014. Interactions between drugs and drug-nutrient in enteral nutrition: a review based on evidences. Nutricion Hospitalaria, 30(3), 514-518.

Seyen, M. V., Colbers, A., Abbink, E. J., Drenth, J. P. H., \& Burger, D. M. 2019. Concomitant Intake of CocaCola to Manage the Drug-Drug Interaction Between Velpatasvir and Omeprazole Studied in Healthy Volunteers. Clinical Pharmacology and Therapeutics, 106(5), 1093-1098.

Zha, W. 2017. Transporter-mediated natural product drug interactions for the treatment of cardiovascular diseases. Journal of Food and Drug Analysis, 26(2S), S32-S44. 


\section{Minicurrículo}

Ana Júlia Carvalho Rocha. Acadêmica do $7^{\circ}$ período do curso de Medicina, Universidade José do Rosário Vellano, Alfenas, Minas Gerais, Brasil.

Ana Rafaela Labouré de Carvalho Vieira. Acadêmica do $7^{\circ}$ período do curso de Medicina, Universidade José do Rosário Vellano, Alfenas, Minas Gerais, Brasil.

André Luiz Oliveira de Faria. Acadêmico do $4^{\circ}$ período do curso de Medicina, Universidade José do Rosário Vellano, Alfenas, Minas Gerais, Brasil.

Guilherme Henrique dos Santos Silva. Acadêmico do $4^{\circ}$ período do curso de Medicina, Universidade José do Rosário Vellano, Alfenas, Minas Gerais, Brasil.

Gérsika Bitencourt Santos. Possui graduação em Farmácia pela UNIFENAS-Alfenas. Mestre e Doutora em Ciências Farmacêuticas pela Universidade Federal de Alfenas (UNIFAL-MG). Docente do curso de Medicina, disciplina de Farmacologia, na Universidade José do Rosário Vellano, Alfenas, Minas Gerais, Brasil.

Como citar: Rocha, A.J.C., Vieira, A.R.L.C., Faria, A.L.O., Silva, G.H.S., \& Santos, G.B. 2021. Interação Fármaco-Nutriente: uma revisão sistemática. Pubsaúde, 6, a140. DOI: https://dx.doi.org/10.31533/pubsaude6.a140

Recebido: 4 mar. 2021.

Revisado e aceito: 25 mar. 2021.

Conflito de interesse: os autores declaram, em relação aos produtos e companhias descritos nesse artigo, não ter interesses associativos, comerciais, de propriedade ou financeiros que representem conflito de interesse.

Licenciamento: Este artigo é publicado na modalidade Acesso Aberto sob a licença Creative Commons Atribuição 4.0 (CC-BY 4.0). 